\title{
Persistent atrial fibrillation: should we always ice the back of the left atrium?
}

\author{
Kavisha Patel $^{1} \cdot$ Jorge Romero $^{1} \cdot$ Luigi Di Biase $^{1}$ (D)
}

Received: 3 June 2019 / Accepted: 18 June 2019 / Published online: 24 July 2019

(C) Springer Science+Business Media, LLC, part of Springer Nature 2019

Two decades ago, a landmark paper identified the pulmonary veins (PVs) as the most frequent trigger source for atrial fibrillation (AF) [1]. Subsequently, pulmonary vein isolation (PVI) emerged as a cornerstone of catheter ablation for AF. However, by itself, PVI is not sufficient to treat persistent atrial fibrillation (persAF). Sustained and non-sustained nonPV triggers have been identified in up to $60 \%$ of cases of catheter ablation of AF [2-4]. These triggers are localized in the posterior wall (PW), superior vena cava, left atrial appendage, coronary sinus, inter-atrial septum, crista terminalis, and vein of Marshall [5, 6]. These areas also serve as substrates for perpetuation of AF. The PW is an embryological "sibling" of the pulmonary veins [7] and its ablation has emerged as an attractive conjunctive strategy with PVI for persAF.

Progressive remodeling within the PW is believed to enrich the pathological substrate for persAF. This notion of increased arrhythmogenicity is supported by cellular evidence, which indicated that the PW in patients with persAF had higher incidence of delayed afterdepolarizations, larger late sodium currents, calcium currents leading to increased calcium content of sarcoplasmic reticulum, and smaller inward rectifier potassium currents [8]. Microscopically, a higher degree of lymphonuclear and fatty infiltration along with fibrosis has been observed in the PW in persAF patients [9-11]. This underlying substrate leads to spontaneous trigger activity, which then propels fibrillatory impulses to both atria. PW ablation is thought to eliminate potential re-entry and focal triggers for AF, "debulk" the left atrium (LA), re-enforce PVI, and cause autonomic denervation by ablation of ganglionated plexi. Multiple strategies have been attempted in the past to achieve PW isolation (PWI). Surgical Cox maze procedure with creation of a box lesion on the PW [12], a "hybrid

Luigi Di Biase

dibbia@gmail.com

1 Division of Cardiology, Albert Einstein College of Medicine at Montefiore Hospital, 111 East 210th St, Bronx, NY, USA approach" [13] with initial thoracoscopic access followed by transvenous catheter ablation to complete endocardial PVI/ PWI were both noted to have significantly lower recurrence rates of atrial arrhythmias. After PVI, creation of linear ablation lines on the roof and floor of LA, connecting the superior and inferior PVs is another approach.

A few small retrospective studies have assessed the incremental value of PWI in addition to PVI to reduce recurrence rates of AF. Despite the growing evidence supporting PWI [14], this adjunctive strategy has not been fully adopted worldwide. The long-term clinical outcomes of PWI in patients with non-paroxysmal AF have been controversial. Two randomized controlled trials failed to prove any benefit of PWI in addition to PVI. It is unclear whether these studies were negative simply because the authors performed a single ring or a box in order to isolate the PW $[15,16]$. Specifically, there may be concern for recurrence of atrial arrhythmias (i.e., microreentry atrial tachycardia or atrial flutter) from reconnections and gap formation within the ablation site, deterioration of atrial mechanical function, and esophageal heating given its proximity to the ablation lesions.

A meta-analysis corroborated that patients who underwent PWI+PVI had lower recurrence of atrial arrhythmias, mainly driven by decrease in AF, than patients who underwent PVI only [17]. The studies included in these meta-analyses had a significant amount of heterogeneity in terms of the technique used for PWI. The most optimal method to successfully accomplish PWI still remains controversial. We currently perform two lines connecting the superior and inferior PVs. Subsequently, high-output pacing $(20 \mathrm{~mA} / 2 \mathrm{msec})$ is performed inside the PW. If myocardial capture is present (i.e., epicardial connections), additional radiofrequency energy is delivered until un-excitability is achieved.

A recent multicenter, randomized trial performing PVI demonstrated non-inferiority of cryoballoon to radiofrequency ablation (RFA) [18]. Prior literature for PWI has mostly utilized point-by-point RFA and the use of cryoballoon for this purpose has been limited. Traditionally, the use of cryoballoon 
ablation has been guided by occlusion of the PVs. However, this is not mandatory and studies have reported optimal lesion creations even with large-sized pulmonary vein ostia when occlusion is not achieved [19,20]. Cryoablation lesions delivered by second-generation balloons are generally large [21] and durable [22], making this a conceptually appealing strategy to isolate the PW.

In a pivotal multicenter retrospective study, Aryana et al. [23] reported outcomes on 390 patients with persAF that underwent index AF ablation with a $28-\mathrm{mm}$ second-generation cryoballoon (222 patients (PWI+PVI), 168 patients (PVIonly)). Cryoablation lesions in the PW were created in a way that their edges overlapped. PWI required an average of $\sim 14$ applications or $\sim 34$ min of cryoablation. Average procedure time was double in the PWI+PVI group as opposed to PVI alone (188 min vs $97 \mathrm{~min}$ ). Non-randomized, chronological design for enrollment was one of the major limitations of this study. Despite this and the need for RFA in more than a third of cases, it highlighted that PWI+PVI using cryoablation could be completed in a safe and efficacious manner, leading to higher freedom from recurrent atrial arrhythmias at 1-year follow-up.

The use of a $28-\mathrm{mm}$ cryoballoon in index ablation is also evaluated in a non-randomized study of 100 patients with persAF [24]. It demonstrated a significantly higher rate of freedom from atrial arrhythmias in the PVI+PW ablation group than in the PVI-only group. Their technique of PWI differed from Aryana et al. [23], where a LA roof block line and bottom block line were constructed for PW ablation. Despite slightly longer total procedural time (average, $192 \mathrm{~min}$ vs $188 \mathrm{~min}$ ), PWI was achieved in only $58 \%$ of patients with this technique. Interestingly, there was no significant difference in the recurrence of atrial arrhythmias between patients who did and did not achieve PWI.

Iacopino et al. [25] in the current issue of JICE assessed the benefit of PWI with cryoablation in addition to redo PVI in patients with recurrent AF. Out of 33 patients undergoing redo ablation, $91 \%$ of patients had successful PWI with cryoablation alone with the remaining patients requiring adjunct RFA. Although limited by a lack of control redo PVIonly group, 1-year freedom from arrhythmia rate was $\sim 85 \%$ without the necessity for additional ablation or Class I or IIII anti-arrhythmic drugs. This was in contrast to the studies mentioned above [23-26], where PWI was performed in the index procedure itself.

Tamborero et al. [16] reported that PWI in addition to PVI did not improve outcomes of catheter ablation for AF. It is important to understand the differences in technique. RFA was used to create linear roof and floor lines. This study noted AF recurrence in majority of PWI patients, with $\sim 70 \%$ of patients with evidence of conduction across roofline or electrical conduction across PW area. A single continuous lesion is difficult to create; even a single gap can lead to reconnection of PW with the rest of the LA. Previous studies have been limited by the fact that the benefit of PWI has been judged by "clinical recurrence of AF" without confirming true isolation of PW.

The following major concerns have been raised for use of cryoablation for PWI. Esophageal injury with formation of atrial-esophageal fistula is the most feared complication of $\mathrm{AF}$ ablation. It is recommended to perform AF cryoablation with continuous esophageal temperature monitoring. With LET (luminal esophageal temperature) monitoring, immediate termination of lesion application for LET temperature $<15{ }^{\circ} \mathrm{C}$ is usually recommended. While follow-up data regarding evidence of esophageal injury post-ablation is limited, Nishimura et al. [24] reported mild erosive esophageal lesions in 3 out of 50 patients that underwent cryoablation. PW mechanical function has been questioned after extensive ablation in the past. However, LA contractility is primarily a function of the anterior muscular portion of the LA with negligible contribution from the PW. No deterioration in LA function was reported after extensive PWI in a prior study [27].

Should PW ablation be performed in all patients with persAF? We believe that PW should definitely be isolated during the index procedure in these patients, particularly if they are obese or have sleep apnea, low left ventricular ejection fraction, hypertrophic cardiomyopathy, mechanical or bioprosthetic mitral valves, and severe LA scarring, since they tend to have a higher prevalence of non-PV triggers [28]. PIVoTAL, a prospective randomized, multicenter trial (ClinicalTrials.gov identifier: NCT03057548) is currently under way to analyze the effect of PWI+PVI as opposed to PVI only using cryoballoon for recurrence of atrial arrhythmias. Overall, the above data suggests that PWI+PVI using cryoballoon can be achieved in a safe and effective manner and provides a high rate of freedom from atrial-tachyarrhythmia in persAF patients. Secondgeneration "cryoballoon" has been used with a reasonable degree of success for PWI, and "icing the back" seems like an appealing strategy in this patient population.

\section{Compliance with ethical standards}

Conflict of interest Dr. Di Biase is a consultant for Biosense Webster and Stereotaxis and has received speaker honoraria/travel from Biosense Webster, St. Jude Medical (now Abbott), Boston Scientific, Medtronic, Biotronik, Pfizer, and Bristol Meyers Squibb. The remaining authors have no disclosures. 


\section{References}

1. Haissaguerre M, Jais P, Shah DC, et al. Spontaneous initiation of atrial fibrillation by ectopic beats originating in the pulmonary veins. N Engl J Med. 1998;339(10):659-66.

2. Arruda M, Mlcochova H, Prasad SK, et al. Electrical isolation of the superior vena cava: an adjunctive strategy to pulmonary vein antrum isolation improving the outcome of AF ablation. J Cardiovasc Electrophysiol. 2007;18(12):1261-6.

3. Chang HY, Lo LW, Lin YJ, et al. Long-term outcome of catheter ablation in patients with atrial fibrillation originating from nonpulmonary vein ectopy. J Cardiovasc Electrophysiol. 2013;24(3):250-8.

4. Santangeli P, Marchlinski FE. Techniques for the provocation, localization, and ablation of non-pulmonary vein triggers for atrial fibrillation. Heart Rhythm. 2017;14(7):1087-96.

5. AlTurki A, Marshall HJ, Proietti R. Targeting nonpulmonary vein triggers during atrial fibrillation ablation: is the game worth the candle? Curr Opin Cardiol. 2018;33(1):50-7.

6. Zhao Y, Di Biase L, Trivedi C, et al. Importance of non-pulmonary vein triggers ablation to achieve long-term freedom from paroxysmal atrial fibrillation in patients with low ejection fraction. Heart Rhythm. 2016;13(1):141-9.

7. Abdulla R, Blew GA, Holterman MJ. Cardiovascular embryology. Pediatr Cardiol. 2004;25(3):191-200.

8. Suenari K, Chen YC, Kao YH, Cheng CC, Lin YK, Chen YJ, et al. Discrepant electrophysiological characteristics and calcium homeostasis of left atrial anterior and posterior myocytes. Basic Res Cardiol. 2011;106(1):65-74.

9. Rohr S. Arrhythmogenic implications of fibroblast-myocyte interactions. Circ Arrhythm Electrophysiol. 2012;5(2):442-52.

10. Wilber DJ. Fibroblasts, focal triggers, and persistent atrial fibrillation: is there a connection? Circ Arrhythm Electrophysiol. 2012;5(2):249-51.

11. Platonov PG, Mitrofanova LB, Orshanskaya V, Ho SY. Structural abnormalities in atrial walls are associated with presence and persistency of atrial fibrillation but not with age. J Am Coll Cardiol. 2011;58(21):2225-32.

12. Voeller RK, Bailey MS, Zierer A, Lall SC, Sakamoto SI, Aubuchon $\mathrm{K}$, et al. Isolating the entire posterior left atrium improves surgical outcomes after the Cox maze procedure. J Thorac Cardiovasc Surg. 2008;135(4):870-7.

13. Pison L, La Meir M, van Opstal J, Blaauw Y, Maessen J, Crijns HJ. Hybrid thoracoscopic surgical and transvenous catheter ablation of atrial fibrillation. J Am Coll Cardiol. 2012;60(1):54-61.

14. Bai R, Di Biase L, Mohanty P, et al. Proven isolation of the pulmonary vein antrum with or without left atrial posterior wall isolation in patients with persistent atrial fibrillation. Heart Rhythm. 2016;13(1):132-40.

15. Lim TW, Koay CH, See VA, McCall R, Chik W, Zecchin R, et al. Single-ring posterior left atrial (box) isolation results in a different mode of recurrence compared with wide antral pulmonary vein isolation on long-term follow-up: longer atrial fibrillation-free survival time but similar survival time free of any atrial arrhythmia. Circ Arrhythm Electrophysiol. 2012;5(5):968-77.

16. Tamborero D, Mont L, Berruezo A, et al. Left atrial posterior wall isolation does not improve the outcome of circumferential pulmonary vein ablation for atrial fibrillation: a prospective randomized study. Circ Arrhythm Electrophysiol. 2009;2(1):35-40.
17. He X, Zhou Y, Chen Y, Wu L, Huang Y, He J. Left atrial posterior wall isolation reduces the recurrence of atrial fibrillation: a metaanalysis. J Interv Card Electrophysiol. 2016;46(3):267-74.

18. Kuck KH, Brugada J, Furnkranz A, et al. Cryoballoon or radiofrequency ablation for paroxysmal atrial fibrillation. N Engl J Med. 2016;374(23):2235-45.

19. Heeger CH, Tscholl V, Wissner E, Fink T, Rottner L, Wohlmuth P, et al. Acute efficacy, safety, and long-term clinical outcomes using the second-generation cryoballoon for pulmonary vein isolation in patients with a left common pulmonary vein: a multicenter study. Heart Rhythm. 2017;14(8):1111-8.

20. Stroker E, Takarada K, de Asmundis C, et al. Second-generation cryoballoon ablation in the setting of left common pulmonary veins: procedural findings and clinical outcome. Heart Rhythm. 2017;14(9):1311-8.

21. Nagashima K, Okumura Y, Watanabe I, et al. Hot balloon versus cryoballoon ablation for atrial fibrillation: lesion characteristics and middle-term outcomes. Circ Arrhythm Electrophysiol. 2018;11(5): e005861. https://doi.org/10.1161/CIRCEP.117.005861.

22. Reddy VY, Sediva L, Petru J, et al. Durability of pulmonary vein isolation with cryoballoon ablation: results from the Sustained PV Isolation with Arctic Front Advance (SUPIR) Study. J Cardiovasc Electrophysiol. 2015;26(5):493-500.

23. Aryana A, Baker JH, Espinosa Ginic MA, Pujara DK, Bowers MR, O'Neill PG, et al. Posterior wall isolation using the cryoballoon in conjunction with pulmonary vein ablation is superior to pulmonary vein isolation alone in patients with persistent atrial fibrillation: a multicenter experience. Heart Rhythm. 2018;15(8):1121-9.

24. Nishimura T, Yamauchi Y, Aoyagi H, Tsuchiya Y, Shigeta T, Nakamura R, Yamashita M, Asano M, Nakamura T, Suzuki H, Shimura T, Kurabayashi M, Keida T, Sasano T, Hirao K, Okishige K. The clinical impact of the left atrial posterior wall lesion formation by the cryoballoon application for persistent atrial fibrillation: feasibility and clinical implications. J Cardiovasc Electrophysiol. 2019;30(6):805-814.

25. Iacopino S, Paparella G, Capulzini L, Ströker E, Beckers S, Osório $\mathrm{TG}$, et al. Posterior box isolation as an adjunctive ablation strategy during repeat ablation with the second-generation cryoballoon for recurrence of persistent atrial fibrillation: 1-year follow-up. J Interv Card Electrophysiol. 2019. https://doi.org/10.1007/s10840-01900551-w.

26. Kuniss M, Greiss H, Pajitnev D, et al. Cryoballoon ablation of persistent atrial fibrillation: feasibility and safety of left atrial roof ablation with generation of conduction block in addition to antral pulmonary vein isolation. Europace. 2017;19(7):1109-15.

27. Verma A, Kilicaslan F, Adams JR, et al. Extensive ablation during pulmonary vein antrum isolation has no adverse impact on left atrial function: an echocardiography and cine computed tomography analysis. J Cardiovasc Electrophysiol. 2006;17(7):741-6.

28. Gianni C, Mohanty S, Trivedi C, Di Biase L, Natale A. Novel concepts and approaches in ablation of atrial fibrillation: the role of non-pulmonary vein triggers. Europace. 2018;20(10):1566-76.

Publisher's note Springer Nature remains neutral with regard to jurisdictional claims in published maps and institutional affiliations. 